\title{
Design, management and comissioning of a utility connected microgrid based on IEC 61850
}

\author{
Albert Ruiz-Álvarez, Alba Colet-Subirachs, Oriol Gomis-Bellmunt, Josep Maria Fernández-Mola, \\ Felipe Álvarez-Cuevas-Figuerola, Joaquim López-Mestre, and Antoni Sudrià-Andreu
}

\begin{abstract}
This paper presents an approach to microgrid automation. The microgrid is composed of three different nodes connected to the public electric grid, which are hardware emulated with back-to-back converters. The microgrid is a flexible and weather independent test bed for testing different control algorithms. The intelligence of the automation system is split in three hierarchical layers: the RMU layer, the iNode layer and the iSocket layer. Communications among iNode and iSockets are according IEC 61850 standard.

The aims of the project is to comission a set of technologies and acquire experience in microgrid automation, IEC 61850 communications and power control algorithms in DER systems.
\end{abstract}

Index Terms-DER systems, IEC 61850, wind power emulator, storage unit emulator, load emulator, back-to-back.

\section{INTRODUCTION}

$\mathbf{T}$ He increased and accelerating load growth is stressing power delivery systems in new ways which they were not designed for. Power systems must benefit from the latest technologies to fulfil the XXI century's electric challenges: assuring electric demand, assuring optimal power quality, integrating renewable energies...

In the recent years there has been a growing interest in the integration of Distributed Energy Resources (DER). DER are small power generators typically located close to loads. Generally speaking, local energy networks increase the independence and improve reliability and efficiency.

DER systems do not mean a specific technology but a new paradigm of the power industry, referring to the management. To date, passive operation of distribution networks has been sufficient in ensuring stable network operation, based upon transmission-level centralised generation delivering power through the distribution network to the end consumer. Active

A. Ruiz-Álvarez, A. Colet-Subirachs, O. Gomis-Bellmunt and A. Sudrià-Andreu are with Catalonia Institute for Energy Research (IREC), Electrical Engineering Area, C/Josep Plà, 2, edifici b2, Planta Baixa, 08019 Barcelona, Spain (e-mail: aruiz@irec.cat, acolet@irec.cat, ogomis@irec.cat and asudria@irec.cat).

J.M. Fernàndez-Mola, O. Gomis-Bellmunt and A. Sudrià-Andreu are with Centre d'Innovació Tecnològica en Convertidors Estàtics i Accionaments (CITCEA-UPC), Departament d'Enginyeria Elèctrica, ETS d'Enginyeria Industrial de Barcelona (ETSEIB), Universitat Politècnica de Catalunya (UPC), Avda. Diagonal, 647, Planta 2, 08028 Barcelona, Spain (e-mail: josep.maria.fernandez@citcea.upc.edu, gomis@citcea.upc.edu and sudria@citcea.upc.edu).

F. Álvarez-Cuevas-Figuerola is with Endesa Servicios S.L., Av. Paralelo, 51 - 08004 Barcelona, Spain (e-mail: felipe.alvarezcuevas@endesa.es).

J. López-Mestre is with Control Intel-ligent de l'Energia (CINERGIA), C/Llull, 51-55, $1^{e} 2^{a}, 08005$ Barcelona, Spain (e-mail: cinergia@cinergia.coop).

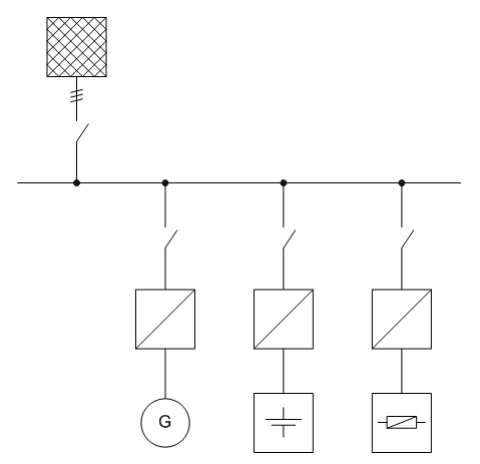

Fig. 1. Conceptual diagram of the microgrid.

Network Management(ANM) solutions are being developed to enable the increasing connection of DERs [1].

The Catalonia Institute for Energy Research (IREC) has developed a low voltage microgrid test bed connected to the public electric grid. Based on peer-to-peer and plug-and-play concepts [2], it is composed of a wind generation node, an energy storage node and a variable load node (Fig. 1).

The automation system is 3-layered. At the top layer, the Remote Management Unit (RMU) manages the overall microgrid. iNode and iSockets controllers, which represent middle and bottom layers respectively, deal with active and reactive power of the nodes of the microgrid and their electric security. Communications among the iNode and the iSockets are according to IEC 61850 . iSockets communicate with power equipment via CAN.

Because all nodes are hardware emulated with back-to-back converters, the microgrid is a useful, flexible and weather independent framework for testing overall control and management algorithms with real power.

This project is the first step of a more complex one. Progressively, the microgrid will be increased up to 19 nodes. The future microgrid will include real wind and photovoltaic generators, real batteries, supercapacitors and real loads, some of which will be electric vehicle chargers [3].

\section{ENDESA'S SMARTCITY PROJECT}

The new energy requirements that will be necessary in the next years will demand a change in the current paradigm for electrical grids. The need for integrating a higher level of renewable resources, the imminent arrival of electric vehicles, and the possibility of actively control all the devices connected to the grid are some examples of these new challenges. And 


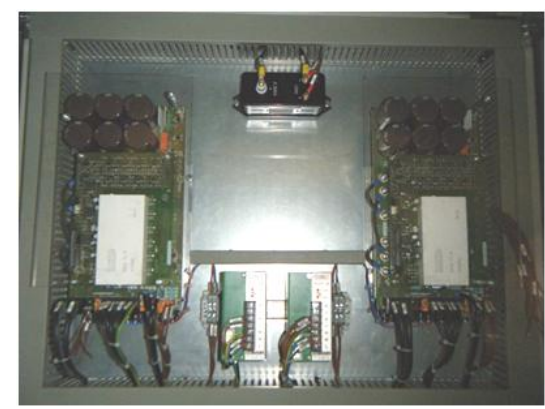

Fig. 2. Converter and Emulator in back-to-back configuration.

Endesa, the largest electric utility company in Spain, is ready to face them all.

Endesa is developing a practical demonstration of smart grid related technologies in the city of Málaga, at the south of Spain. The general vision of the project can be summarized in the following sentence "The energy and environmental needs in the future will demand to optimize the electricity distribution infrastructure. For this reason, the network structure must be reversed. From a few of huge generators to many of small ones". Particular vision involves deploying an exemplary distribution network that includes an heterogeneous mixture of generation and consumption. This will require intercommunication with all electrical infrastructures through communications with the appropriate levels of reliability and bandwidth.

From an electrical point of view, the concept of smart grid is supported in the following three key concepts:

- Advanced Metering Infrastructure (AMI)

- Distributed Energy Resources (DER)

- Advanced Distribution Automation (ADA)

These three systems cannot be considered separately since they are going to share the same infrastructures, and they are intimately related. It is possible to consider that these are three parallel systems sentenced to coexist together.

Endesa, as one of the partners of IREC, has shown a big interest during the development phase of the microgrid. Their interest basically lies in the control algorithms and fault self-healing of the prototype to acquire experience for their project in Málaga.

\section{WORKING SCENARIO: EMULATING GENERATORS AND LOADS}

The microgrid consists of a wind generation node, an energy storage node and a variable load node. The most significant feature of this microgrid is that all nodes are hardware emulated and are capable of injecting/cosuming real power. Each node is composed of two identical bidirectional AC/DC converters in back-to-back configuration (Fig. 2). Converters can work as active rectifier or as active inverter, and are connected to the grid through AC coils (Fig. 3 and Fig. 4).

For every node, one of the converters is programmed to control the maximum active power (variable or non-variable) that can be transmitted accross the DC bus. This power control makes possible the emulation of the behaviour of a wind

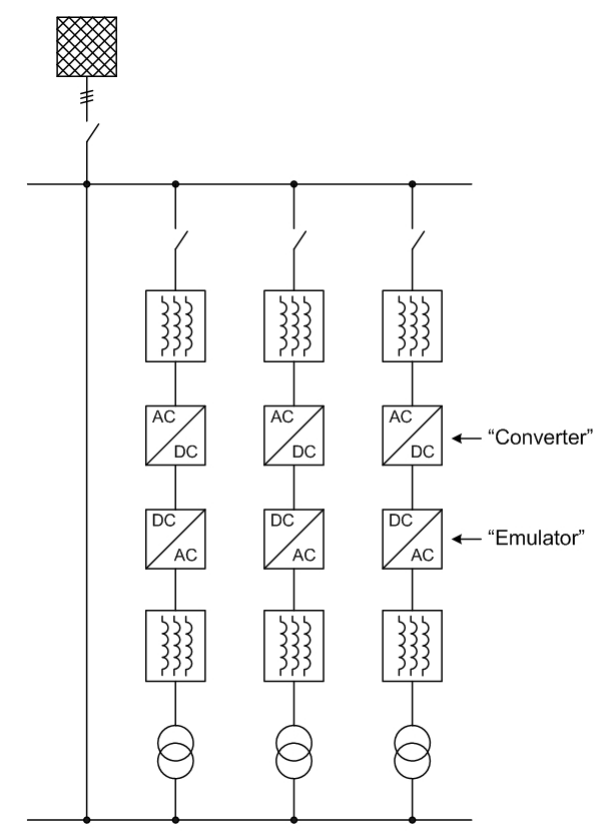

Fig. 3. Electric unifilar diagram of the micogrid.

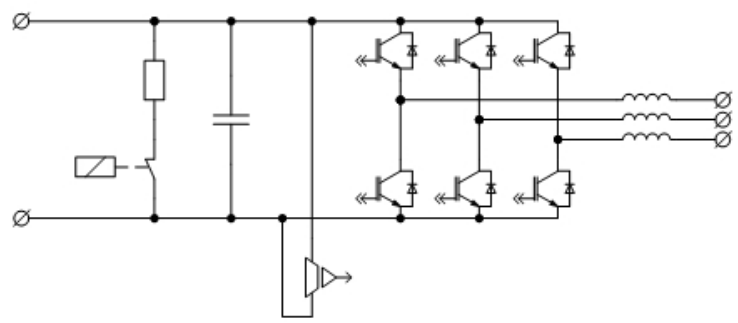

Fig. 4. Simplified electric scheme of the converter.

generator, a photovoltaic generator, a battery, a supercapacitor, a variable load, etc. In this paper, this converter will be referred to as Emulator. The other one will be referred to as Converter.

The Converter can inject or consume power from the grid:

- When injecting (Figure 5.a), the node behaves as a generator. In this situation, the Emulator drives the power from the grid to the DC bus.

- When consuming (Figure 5.b), the node behaves as a load. In this situation, the Emulator drives the power from the DC bus to the grid.

\section{INODE AND ISOCKETS CONTROLLERS}

The microgrid is remotely managed by the RMU. For the time being, the RMU is not a $100 \%$ automatic management system but a manned management system. Tasks performed by the RMU are:

1) To connect the nodes to the electrical grid and to disconnect them.

2) To select the management mode (e.g. centralized, distributed).

3) To command the reference values needed (e.g. active and reactive power to deliver, energy price). 


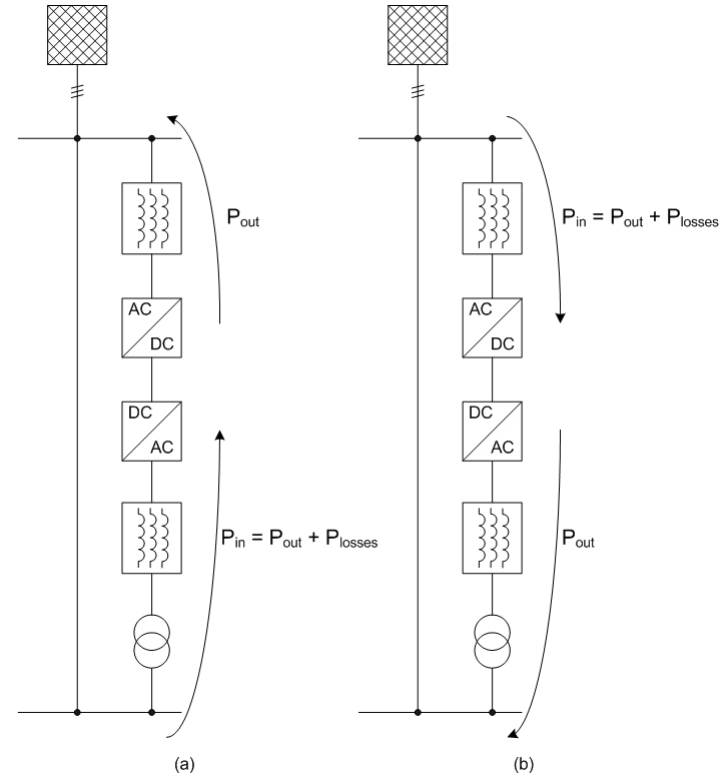

Fig. 5. Power flow. (a) Generation emulation. (b) Consumption emulation.

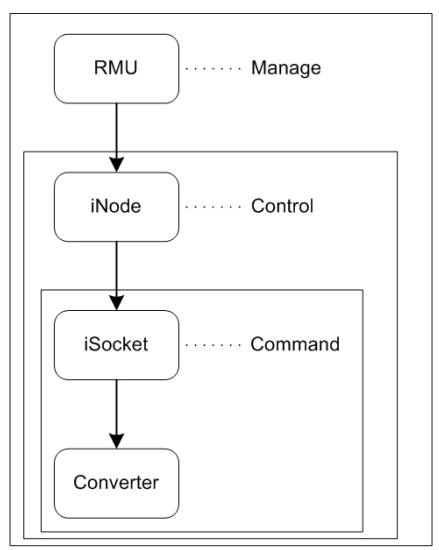

Fig. 6. 3-layered automation system. Roles performed in each layer.

Communications with the nodes are time critical. Because the RMU may be located far away from the microgrid, it is preferable that some management tasks are assumed by nearer devices: iNode and iSockets controllers (Fig. 6).

iNode and iSockets are Intelligent Electronic Devices (IEDs) that perform:

1) Tasks precising a very fast time response (e.g. electric security and stability).

2) Tasks implying high information exchange with the nodes (e.g. execution of active and reactive power control algorithms).

iNode receives RMU's commands (Fig. 7), which set the management mode (centralized or distributed) and the active and reactive power delivered by the overall microgrid. The main functions of the iNode are:

1) To control active and reactive power delivered by the microgrid.

2) To guarantee the security and electrical stability of the microgrid.

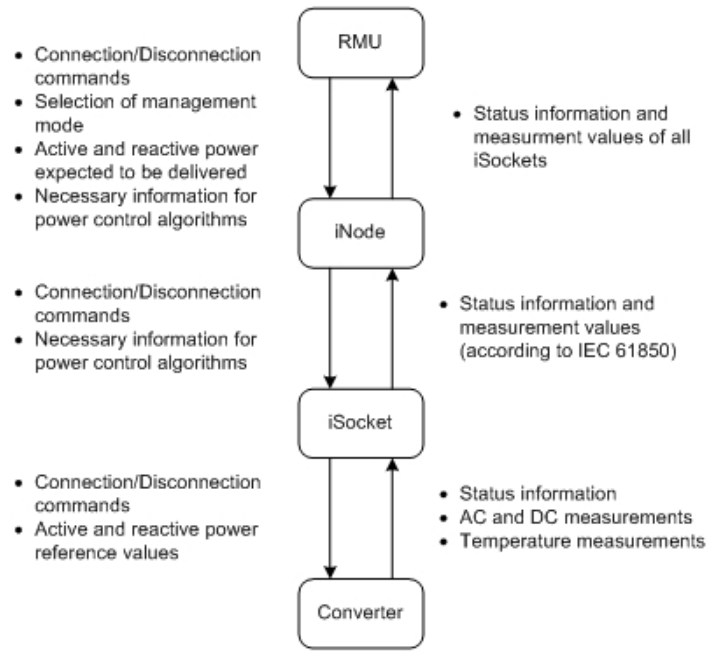

Fig. 7. Simplified information exchange diagram.

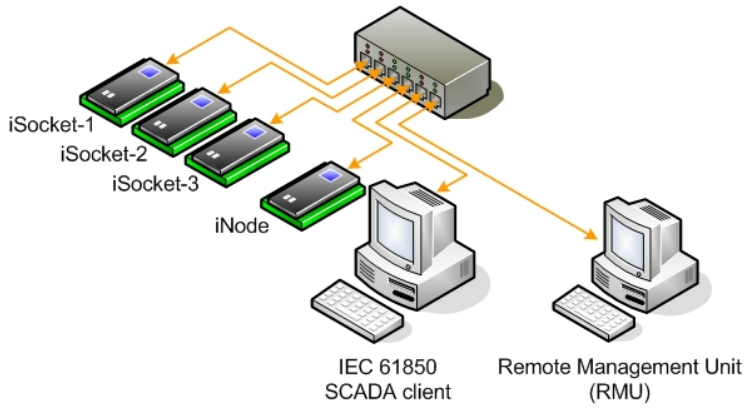

Fig. 8. Interconnection of RMU, iNode, iSockets and SCADA client.

3) To concentrate the microgrid's relevant information and make it visible to the RMU.

For every node there is one iSocket. The iSocket is an interface between the iNode and the Converter (Fig. 7), functions of which are:

1) To command the Converter the active and reactive power expected to be delivered.

2) To guarantee the security of the node.

3) To concentrate the node's relevant information and make it visible to the iNode.

RMU, iNode and iSockets are network connected within the same LAN, based on Ethernet over twisted pair cabling technologies (Fig. 8). The RMU sets a Telnet connection with the iNode, accesses to the command-line interface of the OS and starts the application. Via this virtual terminal connection, the RMU commands the iNode. Communications among iNode and iSockets are Ethernet based according to IEC 61850 standard. iSockets communicate with the Converters via CAN (Fig. 9).

Functions of iNode and iSockets are performed by embedded control boards (Fig. 9 and Fig. 10) with the following characteristics:

- ARM9 based microcontroller, $180 \mathrm{MHz}$

- 32MByte flash memory

- 32MByte SDRAM memory 


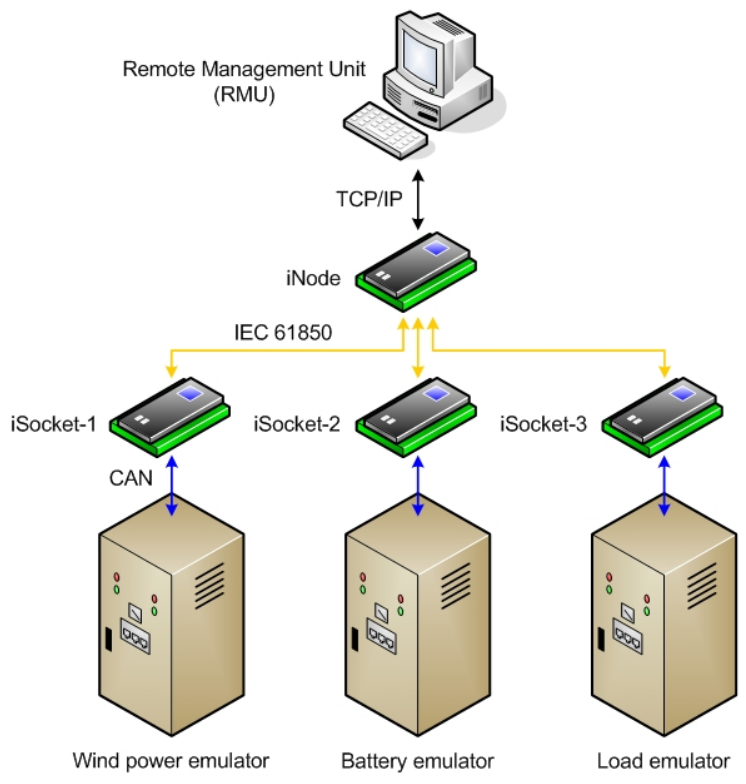

Fig. 9. Communications diagram of the microgrid.

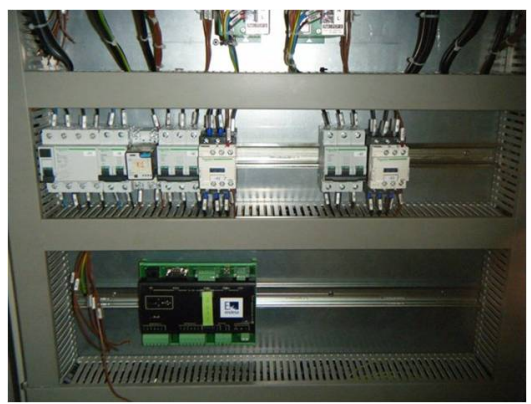

Fig. 10. Embedded control board used for the iNode and the iSockets.

- Isolated 10/100 Base-T Ethernet

- Isolated CAN

- RTC

- 12 isolated digital inputs

- 2 isolated digital outputs

An embedded GNU/Linux based Operating System (OS) has been installed in the microcontroller. Based on the Kernel 2.6, this OS includes drivers for the SDRAM memory, the flash memory, Ethernet, GPIOs, the watchdog timer and the RTC. It has been developed a specific module for CAN communications.

\section{IEC 61850 STANDARD}

In order to ease the engineering and integration effort in the design of the microgrid, communications among iNode and iSockets are based on IEC 61850 International Standard.

IEC 61850 has been elaborated by the ad-hoc group "Substation Control and Protection Interfaces" of IEC Technical Committee 57 for the standardization of communications in Substation Automation Systems (SAS) [4]. With the strong technological development of large-scale integrated circuits, substation secondary equipment rapidly evolved from electro-mechanical devices into digital devices.
Substation Automation Systems (SAS), in turn, started using IEDs to perform the required functions: control, supervision, protection, monitoring, etc.

Generally, IEDs exchange information with other IEDs, i.e. functions exchange information with other functions [5]. Up to now, specific proprietary communication protocols have been used. Since most of them are developed by manufacturers, costly protocol converters are required when using IEDs from different vendors. As a consequence, the need for a standard protocol for efficient communication among IEDs [4] has arisen. The objective of IEC 61850 is to specify requirements and to provide a framework to achieve interoperability among IEDs. This framework specifies the manner that devices should organize information, so it is consistent across all types of devices [6].

The IEC 61850 series approaches the common information found in real devices to abstract information models [7]. All information made available to be exchanged with other devices is defined. The standard also defines the abstract information exchange mechanisms. The major feature of IEC 61850 series is that both information models and information exchange mechanisms are defined in a way that is independent of a concrete implementation.

In IEC 61850, functions are split in smaller entities called Logical Nodes [8]. A Logical Node is a named grouping of data and associated services that is logically related to some power system function [6]. Based on their functionality, a Logical Node contains a list of Data with dedicated Data Attributes [9]. Logical Nodes represent real applications like:

- Distance protection

- Differential protection

- Overcurrent

- Undervoltage

- Transient earth fault

- Measurement

- Metering

- Harmonics and interharmonics

- Switch control

- Circuit breaker

- Battery characteristics

- other

The names of Logical Nodes, Data elements and Data Attributes are determined by the standard. These names are unique and functionally related to the power system purpose. For example, the Logical Node name "XCBR" means Circuit Breaker, the Data element name "Pos" means Position, and the Data Attribute name "ctVal" means Control Value.

Logical Nodes are grouped in Logical Devices. Within each physical device, there are one or more Logical Devices. The IEC 61850 logical device model allows a single physical device to act as a proxy or gateway for multiple devices, providing a standard representation of a data concentrator. The physical device is the device that connects to the network, and is typically defined by its network address.

IEC 61850 defines the Abstract Communication Service Interface (ACSI) [10]. ACSI is a virtual interface to an IED providing abstract communication services (connection, variable access, unsolicited data transfer, device control, etc.) 
including the ones used for exchanging the information represented by the Data and Data Attributes.

The definition of ACSI is independent from any specific underlying protocol. Thus, the IEC 61850 series provides a Specific Communication Service Mapping (SCSM) for mapping ACSI models onto real protocols that are common in the power industry.

IEC 61850-8-1 maps the abstract objects and services to the Manufacturing Message Specification (MMS) protocols [11]. Mapping onto MMS is used for low and medium speed messages, and for file transfer functions. Some ACSI services map onto other protocols, such as Simple Network Time Protocol (SNTP) for time synchronization messages, Generic Object Oriented Substation Event (GOOSE) and Generic Substation Status Event (GSSE) for trips and fast messages. Alternatively, services used for the transmission of sampled values (in which there are raw data messages) are mapped directly into the Ethernet frame [12].

\section{USING IEC 61850 IN IREC'S MICROGRID}

As seen in section IV, only the iNode and the iSockets communicate according to IEC 61850. Functions accomplished by these elements are focused on monitoring and supervising the status of the three nodes of the microgrid, and controlling the active and reactive power of each one. Steps taken towards using this standard for communications were:

1) To identify useful information to implement these functions.

2) To associate this information to the Logical Nodes, Data elements and Data Attributes.

3) To allocate these Logical Nodes in the iNode and the iSockets.

4) To group the Logical Nodes in Logical Devices.

5) To identify services that will be used for Data exchanging.

Fig. 11 shows the Logical Nodes identified for the three power nodes [13].

The main purpose of this project is not the standard exclusively but commissioning a set of technologies. From the very start, three simplifications were considered in IEC 61850 communications:

1) Only mandatory Data elements and Data Attributes would be included.

2) For each power node, Logical Nodes would be grouped in one single Logical Device.

3) Only "Read" and "Write" MMS service would be used for Data exchange.

Of the three, the third simplification is the most relevant. Even though it eases communication software development, these services can only read/write Data Attributes one by one. This implies high communication traffic. To give an example, when the iNode wants to know the three node's active and reactive power measurements, up to $12 \mathrm{TCP} / \mathrm{IP}$ messages are exchanged between the iNode and the iSockets.
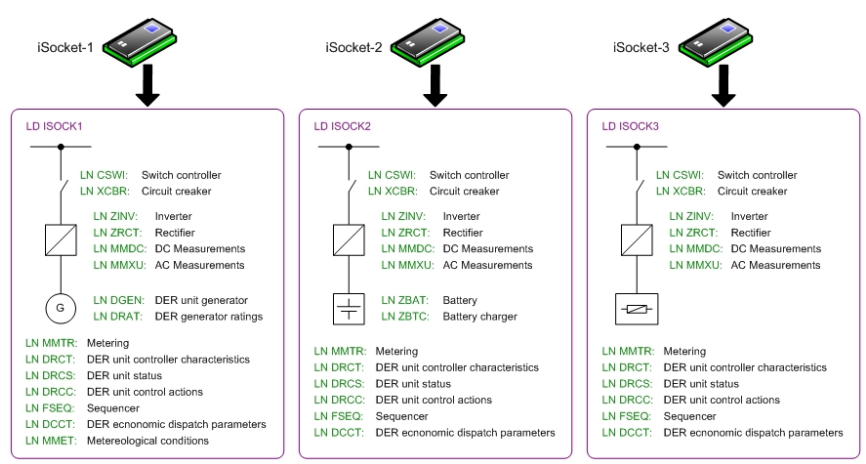

Fig. 11. Logical Nodes and Logical Devices for the three nodes.

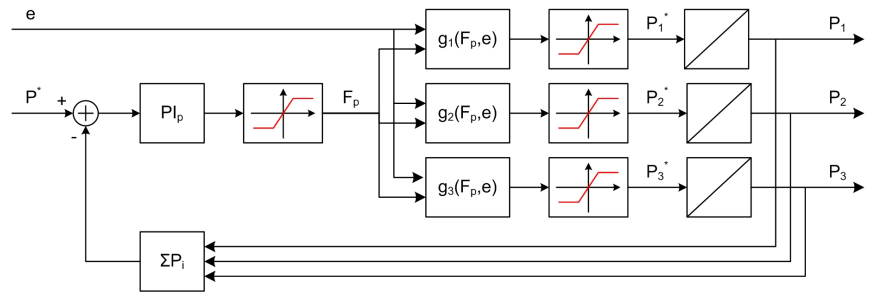

Fig. 12. Active power closed-loop control.

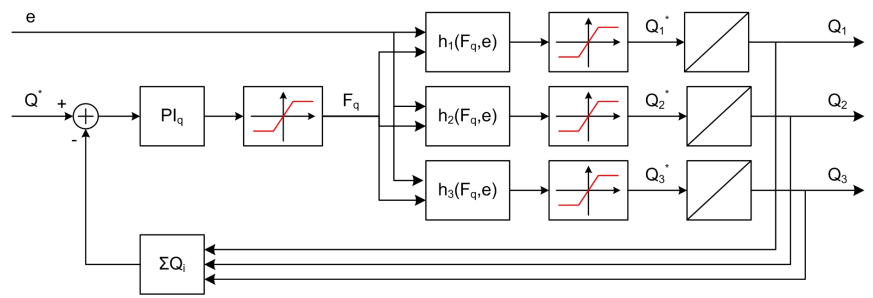

Fig. 13. Reactive power closed-loop control.

\section{MANAGEMENT System, OPERATING MOdeS AND POWER CONTROL ALGORITHMS}

The Management System makes decisions regarding the best use of the generators, load dispatch and energy selling.

In this project, it has been considered that decision of each node's delivered power may be made by either the iNode or the iSockets. Thus, two different operating modes are distinguished:

- Centralized mode. The iNode sets the power of all nodes.

- Distributed mode. Power injection/consumption decisions are made by the iSockets.

\section{A. Centralized mode}

In this operating mode, the iNode controls each node's power so the sum of all powers matches the reference values set by the RMU.

Fig. 12 and Fig. 13 schematize the closed-loop controls for active and reactive power control respectively.

Fig. 14 schematizes the closed-loop operations performed by the iNode for active and reactive power control. Fig. 14 schematizes the operations performed by the iNode for active and reactive power control. As seen in this figure, the inputs and the output of iNode's control are: 


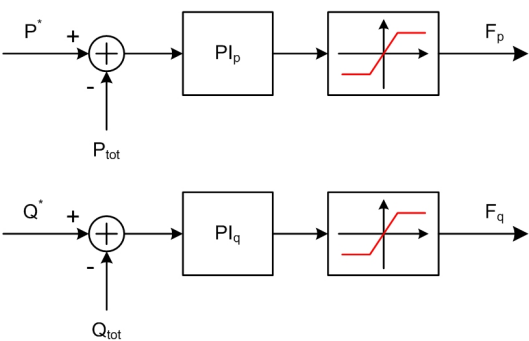

Fig. 14. Functions performed by the iNode for active power control.

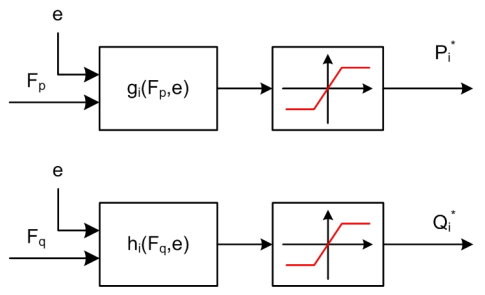

Fig. 15. Functions performed by the iSocket for active power control.

$P^{*}$ Active power reference value $(W)$

$Q^{*}$ Reactive power reference value $(V A r)$

$P_{t o t}$ Overall microgrid's active power $(W)$

$Q_{t o t}$ Overall microgrid's reactive power $(V A r)$

$F_{p}$ Active power control signal

$F_{q}$ Reactive power control signal

$F_{p}$ and $F_{q}$ signals are not power values. In fact, both $F_{p}$ and $F_{q}$ are dimensionless and ranged between:

$$
\begin{aligned}
& -100 \leq F_{p} \leq 100 \\
& -100 \leq F_{q} \leq 100
\end{aligned}
$$

iSockets receive $F_{p}$ and $F q$ signals and the energy price reference. As seen in Fig. 15, from these values iSockets calculate the active and reactive power to command to converters. In this figure:

$e$ Energy price reference (c€)

$P_{i}^{*}$ Active power commanded $(W)$

$Q_{i}^{*}$ Reactive power commanded $(V A r)$

$P_{i}^{*}$ and $Q_{i}^{*}$ functions are piecewise functions defined by (1) and (2) respectively (Fig. 16 and Fig. 17).

$P_{i}^{*}=\left\{\begin{array}{cl}P_{i, M} & F_{p}>\beta_{p i} \\ P_{i, m}+\left(P_{i, M}-P_{i, m}\right) \frac{F_{p}-\alpha_{p i}}{\beta_{p i}-\alpha_{p i}} & \beta_{p i} \geq F_{p} \geq \alpha_{p i} \\ P_{i, m} & F_{p}<\alpha_{p i}\end{array}\right.$

with: $\alpha_{p i} \leq \beta_{p i}$

$Q_{i}^{*}=\left\{\begin{array}{cl}Q_{i, M} & F_{q}>\beta_{q i} \\ Q_{i, m}+\left(Q_{i, M}-Q_{i, m}\right) \frac{F_{q}-\alpha_{q i}}{\beta_{q i}-\alpha_{q i}} & \beta_{q i} \geq F_{q} \geq \alpha_{q i} \\ Q_{i, m} & F_{q}<\alpha_{q i}\end{array}\right.$

with: $\alpha_{q i} \leq \beta_{q i}$

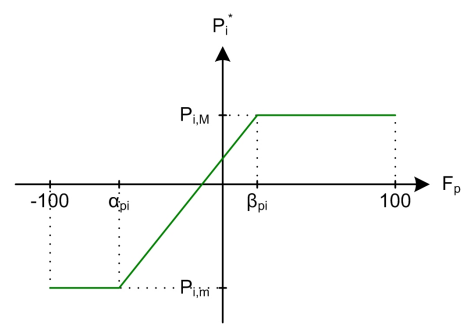

Fig. 16. Graphical representation of $P_{i}^{*}$.

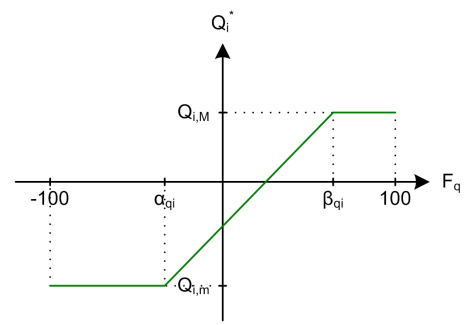

Fig. 17. Graphical representation of $Q_{i}^{*}$.

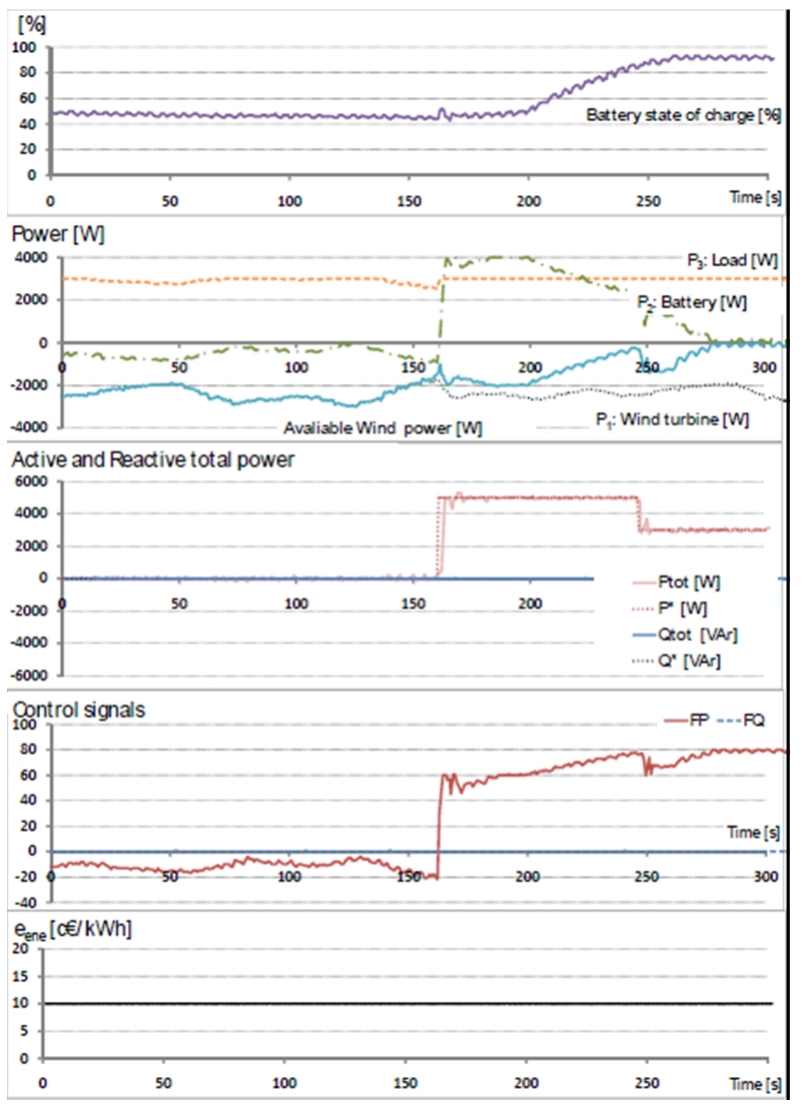

Fig. 18. Real example of microgrid's response in centralized mode.

Independently configured for each node, parameters $\alpha$ and $\beta$ are used to define power limits and participation priorities of all microgrid's units. Depending on the node, these parameters may depend on the generation cost, available stored energy, load reduction cost (among others). Their value is dynamically recalculated.

Fig. 18 illustrates a real evolution of the microgrid after 
changing the active power reference value. Reference [14] analyzes in depth power control algorithms for the centralized mode.

\section{B. Distributed mode}

In this operating mode the iNode has no control on iSockets's power. In fact, there is no feedback.

In the distributed mode, active and reactive power of each node is independent from the other node's ones, and just depends on the energy price reference.

\section{CONCLUSIONS}

In this paper it has been presented a 3-node utility connected microgrid test bed and a hierarchical 3-layered automation system.

The use of hardware emulated nodes makes possible to test management modes and power control algorithms in short-time intervals with total independence from meteorology.

The converters used permit incorporating a real wind generator, a real storage unit and a real load with very few configuration changes. In regard to the management and control software, there would be no changes.

By using IEC 61850 International Standard it is possible to achieve merits such as code reusing, code maintenance and elastic expansibility. MMS services are fast enough for the power control algorithms proposed in this paper. The authors do consider that quantity of messages exchanged for power control should be reduced for the centralized mode. This would decrease the time required for closing active and reactive power control loops, increasing the number of times that control loops are closed in a second. Thereby, stabilization time would be shortened.

\section{REFERENCES}

[1] R. MacDonald, G. Ault, and R. Currie, "Deployment of active network management technologies in the uk and their impact on the planning and design of distribution networks," in Proc. IET-CIRED SmartGrids for Distribution CIRED Seminar, 2008, pp. 1-4.

[2] J. Eto, R. Lasseter, B. Schenkman, J. Stevens, D. Klapp, H. Volkommer, E. Linton, H. Hurtado, and J. Roy, "Overview of the certs microgrid laboratory test bed," in Proc. CIGRE/IEEE PES Joint Symp. Integration of Wide-Scale Renewable Resources Into the Power Delivery System, 2009, p. 1.

[3] M. Roman-Barri, I. Cairo, A. Sumper, and A. Sudria, "Experience on the implementation of a microgrid project in Barcelona," to be published.

[4] IEC 61850 Communication networks and systems in substations - Part 1: Introduction and overview, IEC, First edition, 2003-04 Std.

[5] IEC 61850 Communication networks and systems in substations - Part 5: Communication requirements for functions and device models, IEC, First edition, 2003-07 Std.

[6] R. E. Mackiewicz, "Overview of IEC 61850 and Benefits," in Proc. IEEE PES Power Systems Conf. and Exposition PSCE '06, 2006, pp. 623-630.

[7] IEC 61850 Communication networks and systems in substations - Part 7-1: Basic communication structure for substation and feeder equipment - Principles and models, IEC, First edition, 2003-07 Std.

[8] IEC 61850 Communication networks and systems in substations - Part 7-4: Basic communication structure for substation and feeder equipment - Compatible logical node classes and data classes, IEC, First edition, 2003-05 Std.

[9] IEC 61850 Communication networks and systems in substations - Part 7-3: Basic communication structure for substation and feeder equipment - Common data classes, IEC, First edition, 2003-05 Std.
[10] IEC 61850 Communication networks and systems in substations - Part 7-2: Basic communication structure for substation and feeder equipment - Abstract Communication Service Interface (ACSI), IEC, First edition, 2003-05 Std.

[11] IEC 61850 Communication networks and systems in substations - Part 8-1: Specific Communication Service Mapping (SCSM) - Mappings to MMS (ISO 9506-1 and 9506-2) and to ISO/IEC 8802-3, IEC, First edition, 2004-05 Std.

[12] P. Lloret, J. L. Velasquez, L. Molas-Balada, R. Villafafila, A. Sumper, and S. Galceran-Arellano, "IEC 61850 as a flexible tool for electrical systems monitoring," in Proc. 9th Int. Conf. Electrical Power Quality and Utilisation EPQU 2007, 2007, pp. 1-6.

[13] IEC 61850 Communication networks and systems for power utility automation - Part 7-420: Basic communication structure - Distributed energy resoruces logical nodes, IEC, Edition 1.0, 2009-03 Std.

[14] A. Colet-Subirachs, A. Ruiz-Álvarez, G. Gomis-Bellmunt, F. Alvarez-Cuevas Figuerola, and A. Sudria-Andreu, "Control of a utility conncected microgrid," to be published. 